\title{
Análise do desempenho de subleitos rodoviários à luz de conceitos da Mecânica dos Pavimentos
}

\author{
Analysis of the performance of road subgrades \\ using concepts of Pavement Mechanics
}

Thaís Aquino dos Santos ${ }^{1}$, Rinaldo José Barbosa Pinheiro ${ }^{1}$, Luciano Pivoto Specht ${ }^{1}$, Lélio Antônio Teixeira Brito ${ }^{2}$

\author{
${ }^{1}$ Programa de Pós-Graduação em Engenharia Civil, Universidade Federal de Santa Maria - UFSM, Santa Maria, RS, \\ Brasil. \\ ${ }^{2}$ Programa de Pós-Graduação em Engenharia Civil: Construção e Infraestrutura, Universidade Federal do Rio Grande do \\ Sul - UFRGS, Porto Alegre, RS, Brasil \\ e-mail: thaiis_as@hotmail.com,rinaldo@ufsm.br, luspecht@ufsm.br, leliobrito@gmail.com
}

\section{RESUMO}

O pavimento é considerado um sistema de múltiplas camadas finitas, sujeito às tensões, deformações e deslocamentos provenientes das solicitações cíclicas do tráfego. A fundação da estrutura deve apresentar capacidade de suporte compatível com a intensidade e frequência com a qual será solicitada. Sob essa perspectiva, o presente estudo avalia o desempenho de quatro solos com origens geológicas diferentes, usualmente encontrados em subleitos rodoviários no estado do Rio Grande do Sul, com base em princípios da Mecânica dos Pavimentos. A metodologia aplicada nesse estudo consistiu em simulações numéricas assistidas por dois programas computacionais: AEMC/SisPav e AASHTOWare Pavement ME Design. As análises mecanicistas foram realizadas para avaliar a resposta do solo frente à deformação resiliente e à resistência ao cisalhamento, importantes propriedades no emprego de solos em pavimentação. Os resultados mostraram que a deformação resiliente apresenta um papel preponderante no desempenho dos materiais, visto que, para os solos analisados, não há qualquer indício de ruptura por cisalhamento, tanto como material de subleito rodoviário como fundação de pavimentos de baixo volume de tráfego. Isso, porém não afasta a hipótese de altas deformações plásticas. Quanto à deformação resiliente, observou-se ainda a ineficácia do atual método de dimensionamento de pavimentos flexíveis, que não considera o módulo de resiliência dos materiais de pavimentação, resultando no predomínio de ruptura por fadiga, com exceção do solo denominado de $\mathrm{CB}$ que poderá levar o pavimento a ruptura por excesso de afundamento de trilha de roda.

Palavras-chave: Subleito, análise mecanicista, AASHTOWare Pavement ME Design, AEMC/SisPav.

\begin{abstract}
The pavement is considered a system of multiple finite layers, subject to tensions, deformations and displacements from cyclical traffic demands. The foundation of the structure must have support capacity compatible with the intensity and frequency with which it will be requested. From this perspective, the present study evaluates the performance of four soils with different geological origins, usually found in road subgrades in the state of Rio Grande do Sul, based on the principles of Pavement Mechanics. The methodology applied in this study consisted of numerical simulations assisted by two computational programs: AEMC/SisPav and AASHTOWare Pavement ME Design. The mechanistic analyzes were performed to evaluate the soil response to resilient deformation and shear strength, important properties in the use of paving soils. The results showed that the resilient deformation has a preponderant role in the performance of the materials, since, for the analyzed soils, there is no evidence of shear rupture, both as a road subgrade material and as a foundation of low traffic pavements. This, however, does not rule out the hypothesis of high plastic deformations. Regarding the resilient deformation, it was also observed the inefficiency of the current flexible pavement design method, which does not consider the resilience modulus of the paving materials, resulting in the predominance of fatigue rupture, with the exception of the $\mathrm{CB}$ soil, which could lead to pavement to rupture by excessive wheel track rutting.
\end{abstract}

Keywords: Subgrade, mechanical analysis, AASHTOWare Pavement ME Design, AEMC/SisPav. 


\section{INTRODUÇÃO}

A concepção de uma rodovia está fundamentada em fornecer aos seus usuários uma via segura e confortável ao longo de toda sua vida útil, independentemente da época do ano e sob quaisquer condições climáticas, atendendo as principais demandas funcionais e estruturais [1 - 3]. A estrutura do pavimento é construída com o intuito de distribuir as tensões e deformações induzidas pelo tráfego ao longo de suas camadas, até um nível de intensidade que cada material constituinte dessa estrutura possa suportar [4]. Diz-se que um pavimento falha quando as deformações permanentes são excessivas e/ ou ocorre trincamento demasiado na superfície do revestimento.

O estado de tensões atuante na estrutura como resultado do tráfego é bastante complexo. Um elemento em um pavimento é submetido a pulsos de tensão, que resulta em componentes de tensões vertical, horizontal e de cisalhamento, as quais são transitórias e mudam com o tempo conforme a passagem da roda [5]. Tal estado de tensões depende do comportamento mecânico de cada camada do pavimento e do sistema estratificado formado por essas, principal aspecto estudado pela Mecânica dos Pavimentos [6]. Para SILVA [7], o desafio da Mecânica dos Pavimentos está em compatibilizar o estado de tensões e deformações atuantes no pavimento com o estado de tensões e deformações admissíveis para uma determinada vida de projeto.

$\mathrm{Na}$ engenharia de pavimentos, a utilização de programas computacionais para a análise das tensões e deformações atuantes no sistema em camadas de um pavimento, por meio de soluções da Teoria do Sistema de Camadas Elásticas (TSCE), vem ganhando força, especialmente devido à praticidade no manuseio e alta confiabilidade nos resultados, ampliando a concepção e a aceitação da análise mecanicista no meio técnico e científico [8 - 15]. Isso porque, a partir da determinação dos estados de tensões e deformações em qualquer ponto de análise na estrutura, é possível, através de modelos de desempenho, simular um dano específico acumulado em um determinado pavimento ao longo dos anos. Essa relação constitui o princípio base da abordagem mecanístico-empírica, adotada atualmente por grande parcela dos órgãos rodoviários ao redor do mundo para o dimensionamento de pavimentos flexíveis.

Para YODER e WITCZAK [16], o projeto de pavimentos rodoviários envolve além da análise acerca do desempenho final do sistema frente às inúmeras condições de contorno ao qual o mesmo será submetido, o estudo detalhado de solos e materiais de pavimentação e seus respectivos comportamentos sob cargas repetidas. Como todos os pavimentos derivam do suporte final do subleito, um conhecimento da mecânica básica do solo é essencial. Para os autores, o solo é um material altamente variável, sendo a inter-relação entre a textura do solo, sua densidade, umidade ótima e capacidade de suporte de difícil compreensão, e, em particular, a avaliação do seu comportamento frente aos carregamentos dinâmicos oriundos da circulação de veículos.

Diante do exposto, este trabalho tem como objetivo avaliar o desempenho de solos de subleitos rodoviários comumente utilizados no estado do Rio Grande do Sul, quanto à sua resistência ao cisalhamento e à deformação resiliente, à luz de conceitos e princípios mecanicistas. Além disso, este artigo visa ampliar o uso da análise mecanicista como ferramenta na previsão do desempenho de pavimentos flexíveis e na caracterização do comportamento de materiais empregados na pavimentação, bem como disseminar os conceitos atrelados aos atuais métodos de dimensionamento mecanístico-empírico.

\section{MATERIAIS E MÉTODOS}

De modo a atingir o objetivo geral do estudo, inicialmente foram escolhidos os solos de subleito a serem estudados, tendo como base o seu uso consolidado em obras rodoviárias. Posteriormente, definiu-se uma tipologia de pavimento flexível, que foi dimensionada de acordo com as características de cada material estudado. Por fim, foram realizadas análises mecanicistas, com a finalidade de avaliar a resposta mecânica de cada estrutura dimensionada e a sua relação com as características de resistência ao cisalhamento e deformação resiliente dos solos utilizados na fundação do pavimento.

\subsection{Materiais}

Para compor o estudo, foram definidas quatro jazidas de solos em diferentes regiões do Rio Grande do Sul. A primeira jazida (solo CB) encontra-se localizada na cidade de Coronel Barros, no noroeste do estado. Esse material é oriundo de rochas ígneas basálticas, presente em grande parte do território rio-grandense, constituindo um material altamente argiloso, classificado como latossolo de textura argilosa. A segunda jazida (solo PE) está localizada ao sul do estado, na cidade de Pelotas. Esse solo é resultado do intemperismo de rochas granítico-gnáissicas, com textura média argilosa e horizonte B textural, característico de argissolos vermelho-amarelos, comuns da região. A terceira (solo TR) e quarta jazida (solo RS), encontram-se localizadas no município de Santa Maria, no centro do Rio Grande do Sul, sendo amplamente utilizadas em obras rodoviárias da região, destacando-se a Travessia Urbana de Santa Maria e a duplicação da RS 509, das quais derivam as siglas adotadas. Ambos materiais são provenientes de formações sedimentares da Província Paraná, pedogeneticamente classificados como argissolos vermelho-amarelo e vermelho escuro de texturas areno-argilosa e média argilosa, respectivamente. A Figura 1 apresenta o mapa de localização das jazidas. 
As informações quanto à caracterização dos solos, à granulometria, limites de Atterberg, peso específico real dos grãos, dados de compactação para a energia Proctor Normal, Índice de Suporte Califórnia (ISC) e expansão, coesão (c') e ângulo de atrito interno $(\phi)$, bem como suas classificações MCT (Miniatura, Compactado, Tropical), SUCS e AASHTO estão apresentados na Tabela 1. Os procedimentos metodológicos adotados para esses ensaios estão descritos em SANTOS et al. [15].

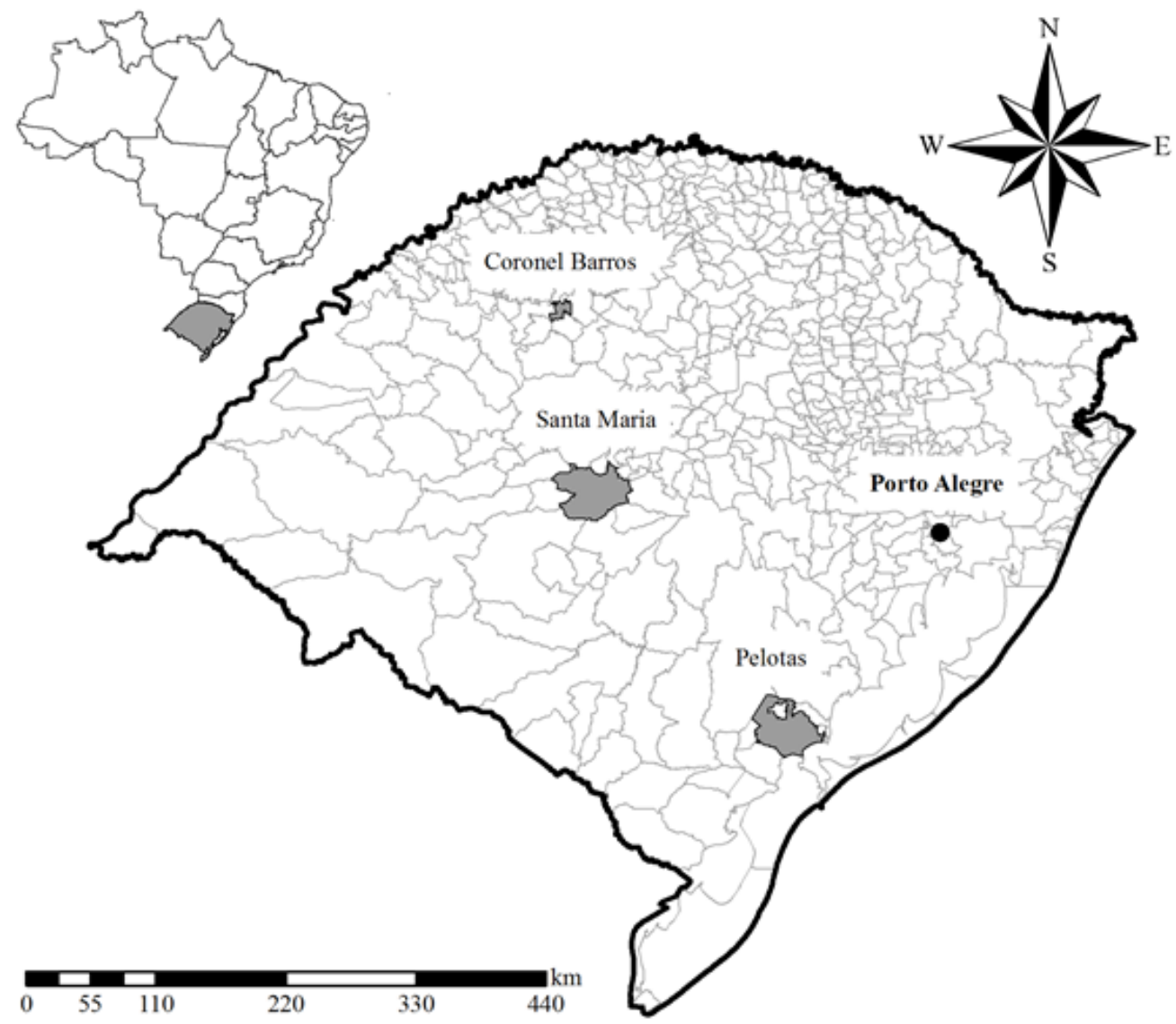

Figura 1: Mapa de localização das jazidas de solo

Tabela 1: Síntese da caracterização dos solos

\begin{tabular}{ccccc}
\hline PARÂMETROS & SOLO CB & SOLO PE & SOLO TR & SOLO RS \\
\hline \% pedregulho $(>2,0 \mathrm{~mm})$ & 0 & 1 & 1 & 2 \\
\% areia grossa $(0,6 \mathrm{~mm}-2,0 \mathrm{~mm})$ & 2 & 5 & 12 & 11 \\
$\%$ areia média $(0,2 \mathrm{~mm}-0,6 \mathrm{~mm})$ & 6 & 15 & 26 & 16 \\
$\%$ areia fina $(0,06 \mathrm{~mm}-0,2 \mathrm{~mm})$ & 5 & 22 & 18 & 15 \\
$\%$ silte $(2 \mu \mathrm{m}-0,06 \mathrm{~mm})$ & 19 & 22 & 13 & 14 \\
$\%$ argila $(<2 \mu \mathrm{m})$ & 68 & 35 & 30 & 41 \\
\hline Limite de Liquidez $-\mathrm{LL}(\%)$ & 56 & 41 & 39 & 47 \\
Limite de Plasticidade $-\mathrm{LP}(\%)$ & 25 & 18 & 20 & 29 \\
Índice de Plasticidade - IP $(\%)$ & 31 & 23 & 19 & 18 \\
\hline Peso específico real dos grãos $\gamma_{\mathrm{s}}\left(\mathrm{kN} / \mathrm{m}^{3}\right)$ & 28,09 & 25,64 & 25,54 & 25,75 \\
\hline Classificação SUCS & $\mathrm{CH}$ & $\mathrm{CL}$ & $\mathrm{SC}$ & CL \\
Classificação AASHTO/TRB & $\mathrm{A}-7-6$ & $\mathrm{~A}-7-6$ & $\mathrm{~A}-6$ & A-7-6 \\
Classificação MCT ${ }^{1}$ & LG & LG' & LG' & LG' \\
\hline
\end{tabular}




\begin{tabular}{|c|c|c|c|c|c|c|}
\hline \multicolumn{3}{|c|}{ PARÂMETROS } & SOLO CB & SOLO PE & SOLO TR & SOLO RS \\
\hline \multirow{8}{*}{$\begin{array}{c}\text { Energia Proctor } \\
\text { Normal }\end{array}$} & \multicolumn{2}{|c|}{ Umidade ótima - wótima (\%) } & 28,7 & 17,1 & 15,1 & 19,9 \\
\hline & \multicolumn{2}{|c|}{$\begin{array}{l}\text { Peso específico aparente seco } \\
\text { máximo }-\gamma_{\mathrm{d}}\left(\mathrm{kN} / \mathrm{m}^{3}\right)\end{array}$} & 14,71 & 17,40 & 17,87 & 16,47 \\
\hline & \multicolumn{2}{|c|}{ ISC (\%) } & 10 & 10 & 14 & 16 \\
\hline & \multicolumn{2}{|c|}{ Expansão (\%) } & 0,24 & 0,27 & 0,27 & 0,18 \\
\hline & \multirow{2}{*}{ wótima } & $\mathrm{c}^{\prime}(\mathrm{kPa})$ & 48,85 & 43,35 & 46,65 & 50,95 \\
\hline & & $\phi\left(^{\circ}\right)$ & 34,80 & 52,56 & 34,11 & 38,33 \\
\hline & \multirow{2}{*}{${ }^{2}$ wótima $+2 \%$} & $\mathrm{c}^{\prime}(\mathrm{kPa}$ & 7,75 & 10,25 & 16,90 & 8,85 \\
\hline & & $\phi\left(^{\circ}\right)$ & 35,94 & 36,04 & 30,92 & 38,65 \\
\hline
\end{tabular}

Nota: ${ }^{1}$ LG': grupo de solos com comportamento laterítico argiloso; ${ }^{2}$ Condição de umidade pós-compactação crítica utilizada nas análises mecanicistas relacionadas à resistência ao cisalhamento.

\subsection{Análises mecanicistas}

Para a realização das simulações numéricas, optou-se por uma estrutura tipicamente utilizada no estado do Rio Grande do Sul, composta por CA (concreto asfáltico) como camada de revestimento, BGS (brita graduada simples) como base, MS (macadame seco) como camada de sub-base e os diferentes solos pesquisados aplicados no subleito do pavimento.

Após a definição da estrutura, o seu dimensionamento foi realizado através do método de dimensionamento de pavimentos flexíveis novos em vigência no Brasil - método do DNIT [17, 18], considerando um período de projeto de 10 anos e um número de solicitações de carga $\left(\mathrm{N}_{\text {PROJETO }}\right)$ de 2,5 $\times 10^{7}$. Esse valor foi extraído de KLAMT [19], baseado nos valores obtidos na contagem da praça de pedágio Retiro, localizada na cidade de Pelotas/RS. Assim, de posse das estruturas dimensionadas, as análises mecanicistas foram realizadas com base em duas propriedades de interesse no âmbito da pavimentação: deformação resiliente e resistência ao cisalhamento.

\subsubsection{Deformação resiliente}

A deformação resiliente dos materiais de pavimentação está diretamente associada a um dos principais mecanismos de ruptura recorrentes em pavimentos flexíveis brasileiros: o trincamento por fadiga [3]. Por esse motivo, o módulo de resiliência é considerado fundamental para a análise e projeto de pavimentos, o qual é amplamente utilizado nos métodos de dimensionamento internacionais. Apesar de não ser considerado no dimensionamento de pavimentos flexíveis novos, segundo as diretrizes presentes no método do DNIT, é de conhecimento geral a importância desse parâmetro no desempenho do pavimento ao longo de sua vida útil de projeto. Tanto que tal parâmetro foi incorporado como peça chave no novo método de dimensionamento nacional - MeDiNa, atualmente em fase final de desenvolvimento. Nesse contexto, o comportamento das estruturas, considerando os efeitos atrelados às características resilientes dos solos de subleito, foi analisado por meio de dois softwares que permitem uma visão geral da resposta mecânica do pavimento submetido aos carregamentos cíclicos: AASHTOWare Pavement ME Design e AEMC/SisPav.

A Tabela 2 apresenta os dados de entrada adotados para ambas simulações. No subleito, foram utilizados os módulos de resiliência não lineares dos solos em estudo, obtidos nos ensaios triaxiais de cargas repetidas realizados por SANTOS et al. [15], uma vez que representam as características de deformabilidade dos solos em função do estado de tensões atuante na estrutura simulada. Os demais parâmetros de entrada referentes aos módulos resilientes e os coeficientes de Poisson seguiram os valores de referência encontrados na literatura e em retroanálises realizadas em rodovias do estado [1, 2, 20 - 22]. Para todas as análises, adotou-se a condição de camadas não aderidas, submetidas ao eixo padrão rodoviário (eixo simples de rodas duplas - ESRD carregado com $80 \mathrm{kN}$ e pressão de inflação dos pneus de $0,56 \mathrm{MPa}$ ).

O AASHTOWare Pavement ME Design é o software que acompanha o método de dimensionamento mecanístico-empírico da AASHTO - MEPDG, sendo considerado atualmente uma das ferramentas mais completas para análise de desempenho estrutural e funcional de pavimentos rígidos ou flexíveis, novos ou reabilitados, utilizando princípios empírico mecanicistas para análise dos dados de clima, materiais empregados em pavimentação, tráfego e estrutura proposta. Seu relatório de saída possibilita avaliar o desempenho das estruturas frente aos diversos mecanismos de ruptura, porém neste trabalho foram analisados somente os mecanismos de fadiga e deformação permanente (ATR). 
Tabela 2: Dados de entrada no AEMC/SisPav e AASHTOWare Pavement ME Design

\begin{tabular}{|c|c|c|c|c|}
\hline CAMADA & MATERIAL & $\begin{array}{l}\text { ESPESSURA } \\
(\mathrm{cm})\end{array}$ & $\begin{array}{c}\text { MÓDULO DE RESILIÊNCIA } \\
(\mathrm{MPa})\end{array}$ & $\begin{array}{l}\text { COEFICIENTE DE } \\
\text { POISSON }\end{array}$ \\
\hline Revestimento & $\mathrm{CA}$ & $*$ & $\begin{array}{c}5000 \\
\text { Default }^{1}\end{array}$ & 0,30 \\
\hline Base & BGS & $*$ & 200 & 0,35 \\
\hline Sub-Base ${ }^{2}$ & MS & $*$ & 300 & 0,35 \\
\hline \multirow{4}{*}{ Subleito } & Solo CB & \multirow{4}{*}{$\infty$} & $\mathrm{M}_{\mathrm{R}}=76,37 \sigma_{3}^{0,311} \sigma_{\mathrm{d}}^{-0,252}$ & \multirow{4}{*}{0,45} \\
\hline & Solo PE & & $\mathrm{M}_{\mathrm{R}}=216,02 \sigma_{3}^{0,254} \sigma_{\mathrm{d}}^{-0,131}$ & \\
\hline & Solo TR & & $\mathrm{M}_{\mathrm{R}}=624,31 \sigma_{3}^{0,644} \sigma_{\mathrm{d}}^{-0,202}$ & \\
\hline & Solo RS & & $\mathrm{M}_{\mathrm{R}}=484,39 \sigma_{3}^{0,488} \sigma_{\mathrm{d}}^{0,065}$ & \\
\hline
\end{tabular}

Nota: * Espessuras de acordo com o dimensionamento de cada estrutura; ${ }^{1}$ No software AASHTOWare foram utilizados os dados de referência presentes no software; ${ }^{2}$ Valores adotados quando houver a camada de sub-base na estrutura.

Os parâmetros de entrada apresentados na Tabela 2 foram inseridos no software, que possibilita ainda incorporar e computar outras características dos materiais empregados em cada uma das camadas do pavimento. Assim, para os solos em estudo, empregados na fundação do pavimento, foram especificados ainda os valores de limites de liquidez e plasticidade, teor ótimo de compactação e suas respectivas classificações AASHTO/TRB. O tráfego foi completamente discretizado, utilizando-se o espectro completo de carga por eixo, para cada tipo de eixo, visto que o AASHTOWare não caracteriza o tráfego em termos de uma carga por eixo equivalente (Equivalent Single Axle Load - ESAL). De forma a atender aos limites de projeto, adotou-se uma confiabilidade de $50 \%$ e um período de projeto de 10 anos. Em relação aos dados climáticos, adotou-se as características da cidade de Savannah, no estado da Geórgia (EUA), devido à semelhança com o clima presente no estado do Rio Grande do Sul. A previsão de desempenho foi realizada utilizando os modelos de previsão e os coeficiente de calibração presentes no próprio software. Assim, ao se utilizar a modelagem específica da ferramenta, foi possível observar o comportamento das estruturas frente aos mecanismos de ruptura por um ponto de vista diferente, com base nos princípios do dimensionamento mecanístico-empírico americano.

O AEMC (Análise Elástica de Múltiplas Camadas) é um programa específico para o cálculo de tensões, deformações e deslocamentos, com rotinas para entrada de dados e apresentação de resultados, presente no software SisPav [8]. Com o auxílio dessa ferramenta, foram obtidas as tensões e deformações atuantes nas estruturas, e a partir de princípios mecanicistas, foi possível avaliar a influência mecânica dos diferentes solos na degradação do pavimento flexível pelo mecanismo de ruptura por fadiga. Optou-se por analisar exclusivamente esse mecanismo de degradação, tendo em vista que o método oficial do DNIT prescreve o dimensionamento de camadas sobrejacentes ao subleito de modo a protegê-lo de uma possível ruptura por excesso de deformação permanente. No entanto, vale ressaltar a importância do estudo da deformação permanente de solos utilizados na pavimentação, principalmente no que tange os avanços dos métodos de dimensionamento mecanístico-empírico.

As deformações de tração na fibra inferior do revestimento $\left(\varepsilon_{\mathrm{t}}\right)$ foram analisadas e os valores admissíveis de solicitações à fadiga foram calculados através do modelo de previsão de desempenho à fadiga desenvolvido pela Federal Highway Administration - FHWA, apresentado na Equação 1.

$$
\mathrm{N}_{\text {AASHTO }}=1,092.10^{-6} \cdot\left(\frac{1}{\varepsilon_{\mathrm{t}}}\right)^{3,512}
$$

Onde $\mathrm{N}$ é o número de solicitações admissíveis ao trincamento por fadiga e $\varepsilon_{\mathrm{t}}$ é a deformação horizontal de tração na fibra inferior do revestimento asfáltico. Para efeitos de comparação com o $\mathrm{N}$ de projeto, adotou-se a relação $\mathrm{N}_{\mathrm{USACE}}=$ 3. $\mathrm{N}_{\text {AASHTO }}$.

Os números de repetições de cargas admissíveis à fadiga $\left(\mathrm{N}_{\mathrm{FADIGA}}\right)$ obtidos para cada estrutura em análise foram comparados com o número de solicitações de carga de projeto $\left(\mathrm{N}_{\text {PROJETO }}\right)$. Nos casos em que a estrutura apresentou ruptura precoce $\left(\mathrm{N}_{\text {FADIGA }}\right.$ inferior ao $\mathrm{N}_{\text {PROJETO }}$ ), aumentou-se a espessura do revestimento com o intuito de atender ao critério de trincamento por fadiga dessa camada. 


\subsubsection{Resistência ao cisalhamento}

Segundo HOLTZ e KOVACS [23], a resistência ao cisalhamento do solo é considerada um dos aspectos mais importantes na engenharia geotécnica, influenciando a capacidade de carga de fundações superficiais e profundas, a estabilidade de encostas, o projeto de estruturas de contenção e, indiretamente, o projeto de pavimentos. Assim, além da deformação resiliente, o desempenho dos solos em campo frente à resistência ao cisalhamento também foi simulado por meio da ferramenta AEMC.

A metodologia da análise em relação à resistência ao cisalhamento aponta algumas semelhanças com a sistemática da análise apresentada anteriormente. As estruturas dimensionadas, bem como os dados de entrada na ferramenta AEMC foram os mesmos. O que diferencia uma análise da outra são os carregamentos adotados e os dados analisados. Foram utilizados três níveis de carregamentos distintos: o eixo simples de rodas duplas (ESRD) carregado com $80 \mathrm{kN}$ (8,2 toneladas), $98 \mathrm{kN}$ (10 toneladas) e $118 \mathrm{kN}$ (12 toneladas), todos com pressão de inflação dos pneus de 0,56 MPa. Foram definidos pontos de análise de $10 \mathrm{~cm}$ em $10 \mathrm{~cm}$ a partir do topo do subleito, até cerca de 2,7 metros de profundidade. Analisando os pares de tensão de cisalhamento e tensão vertical, foi possível a construção de um gráfico, comparando as envoltórias de ruptura obtidas através dos ensaios laboratoriais realizados por SANTOS et al. [15], e as envoltórias construídas a partir da análise mecanicista, para cada um dos carregamentos utilizados. Com isso, as tensões de cisalhamento atuantes nas fundações das estruturas dimensionadas foram comparadas com as tensões cisalhantes de ruptura nas condições de umidade analisadas, verificando se os solos em estudo levam o subleito à ruptura por cisalhamento. Ressalta-se que as condições de umidade analisadas se referem às envoltórias de ruptura construídas na umidade ótima e em amostras submetidas ao umedecimento de $2 \%$ pós-compactação.

Uma análise complementar também foi realizada, com o objetivo de analisar a resposta ao cisalhamento dos materiais quando empregados em subleitos de pavimentos de baixo volume de tráfego. A metodologia seguiu as diretrizes apresentadas anteriormente, diferenciando-se somente pela estrutura analisada. Em todos os casos, o carregamento foi aplicado diretamente sobre o solo de fundação, simulando uma camada delgada de tratamento superficial duplo (TSD) sobre o material. Os dados de entrada, assim como os níveis de carregamento utilizados foram os mesmos. Observou-se que durante a realização da referida análise, as simulações apresentaram peculiaridades e por esse motivo os valores de tensão máxima cisalhante, tensão normal atuante e profundidade (z), onde ocorre o pico da tensão cisalhante, foram calculados através das equações presentes em MEDINA e MOTTA [3], e aqui apresentadas nas Equações 2, 3 e 4, respectivamente.

$$
\begin{gathered}
\tau_{\text {máx }}=\frac{p}{2}\left[\frac{1-2 \mu}{2}+\frac{2}{9}(1+\mu) \sqrt{2(1+\mu)}\right] \\
\sigma_{z}=p\left[1-\frac{z^{3}}{\left(z^{2}+a^{2}\right)^{\frac{3}{2}}}\right] \\
z=a \sqrt{\frac{2(1+\mu)}{7-2 \mu}}
\end{gathered}
$$

Onde $\tau_{\text {máx }}$ é a tensão cisalhante máxima no eixo de simetria, z é a profundidade onde a tensão cisalhante é máxima, $\sigma_{z}$ é a tensão normal atuante à profundidade z no eixo de simetria, $\mathrm{p}$ é a pressão da área circular carregada, é o raio da área carregada e $\mu$ é o coeficiente de Poisson. Cabe salientar que as equações utilizadas não levam em consideração a deformabilidade dos solos estudados, não havendo diferenciação entre eles. Em relação aos níveis de carregamento, o parâmetro que expressa essa distinção é o raio da área carregada, crescente com o aumento da carga.

\section{RESULTADOS E DISCUSSÕES}

\subsection{Dimensionamento das estruturas}

De acordo com as diretrizes apresentadas na metodologia, as estruturas foram inicialmente dimensionadas pelo método empírico do DNIT, baseado nos valores de ISC. Destaca-se que, em alguns casos, a espessura da camada obtida foi inferior ao mínimo presente em norma, adotando-se assim o valor mínimo. A Tabela 3 sintetiza as espessuras das estruturas dimensionadas.

A estrutura E01 foi dimensionada para um ISC de 10\%, referente aos solos CB e PE, e é composta de $10 \mathrm{~cm}$ de 
concreto asfáltico (CA), $15 \mathrm{~cm}$ de brita graduada simples (BGS) e $16 \mathrm{~cm}$ de macadame seco (MS). A estrutura E02 foi dimensionada para o solo TR, com ISC de 14\%, e é composta de $10 \mathrm{~cm}$ de camada de revestimento (CA) e $17 \mathrm{~cm}$ de base granular (BGS). A estrutura E03 (ISC=16\%) é semelhante à estrutura E02, diferenciando-se apenas pela espessura da camada de base, cerca de $15 \mathrm{~cm}$ de brita graduada simples. Tanto a estrutura E02 quanto a estrutura E03 não apresentaram camada de sub-base. Vale ressaltar que, tal como as análises em relação à deformação resiliente, as análises em relação à resistência ao cisalhamento foram realizadas com base nas estruturas aqui apresentadas.

Tabela 3: Estruturas dimensionadas a partir do método de dimensionamento empírico do DNIT baseado no ISC

\begin{tabular}{cccc}
\hline \multirow{2}{*}{ CAMADAS } & \multicolumn{3}{c}{ ESPESSURA DAS CAMADAS } \\
\cline { 2 - 4 } & ESTRUTURA E01 & ESTRUTURA E02 & ESTRUTURA E03 \\
\hline Revestimento & $10 \mathrm{~cm}$ & $10 \mathrm{~cm}$ & $10 \mathrm{~cm}$ \\
\hline Base & $15 \mathrm{~cm}$ & $17 \mathrm{~cm}$ & $15 \mathrm{~cm}$ \\
\hline Sub-Base & $16 \mathrm{~cm}$ & - & - \\
\hline \multirow{2}{*}{ Subleito } & $\infty$ & $\infty$ & $\infty$ \\
& Solo CB/Solo PE & Solo TR & Solo RS \\
\hline
\end{tabular}

\subsection{Deformação resiliente}

A expectativa de vida útil das estruturas, obtida com base nas projeções de comportamento extraídos do AASHTOWare Pavement, é apresentada na Figura 2. O relatório de saída desse software permite observar o comportamento do pavimento frente à fadiga e deformação permanente (relacionada ao excesso de afundamentos em trilha de roda - ATR) ao longo do período de projeto. Para construção do gráfico, apresentado na Figura 2, adotaram-se os seguintes critérios utilizados pelo software: $19 \mathrm{~mm}$ para afundamentos em trilha de roda (ATR) e $25 \%$ de área trincada. Pode-se perceber que, em nenhum dos casos, os pavimentos atingem o tempo de vida útil para o qual foram dimensionados (10 anos). A durabilidade dos pavimentos variou de 5 anos e meio (E01 - solo PE) a 2 anos e 4 meses (E03 - solo RS). Para a maioria das estruturas, o mecanismo de ruptura foi o trincamento por fadiga, com exceção daquela dimensionada para o solo CB. A estrutura E01, adotando o solo CB como subleito, apresentou uma vida útil de cerca de 2 anos e 8 meses, rompendo nesse caso por deformação permanente. Em vista disso, ressalta-se novamente a importância do estudo da deformação permanente em materiais utilizados em pavimentação.

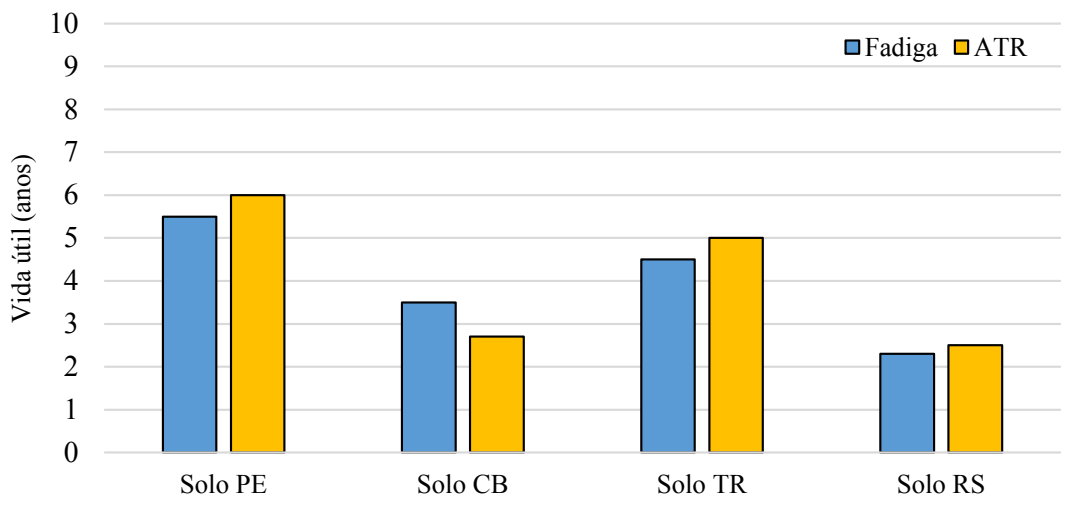

Figura 2: Projeção da vida útil das estruturas a partir da análise realizada no AASHTOWare Pavement ME Design

No Brasil, o trincamento por fadiga predomina, pois há o superdimensionamento dos pavimentos quanto à deformação permanente, dado pelo método empírico do DNIT. Além disso, tal método preconiza uma espessura máxima de $12,5 \mathrm{~cm}$ para um tráfego muito pesado ( $\mathrm{N}>5 \times 10^{7}$ solicitações), sem considerar as características resilientes do material utilizado na camada de revestimento e sua capacidade de resistir aos esforços de solicitações dinâmicas. Em análises mecanicistas realizadas com o AASHTOWare em pavimentos dos Estados Unidos [12, 13], nota-se que o mecanismo de ruptura por deformação permanente é mais suscetível a ocorrer do que a degradação por fadiga. Isso pode estar atrelado às altas espessuras da camada de revestimento, que retarda o aparecimento de fissuras por fadiga, e às mudanças climáticas associadas ao fenômeno de gelo/degelo que pode intensificar o aparecimento de deformações plásticas excessivas.

A segunda análise consistiu na simulação numérica das estruturas dimensionadas, por meio da ferramenta AEMC, avaliando se o método de dimensionamento oficial do DNIT impede a ruptura do pavimento pelo excesso de trincas por 
fadiga. A Tabela 4 apresenta as deformações horizontais de tração na fibra inferior do revestimento $\left(\varepsilon_{t}\right)$, assim como o número de solicitações admissíveis à fadiga para cada uma das estruturas analisadas. Observando os resultados apresentados, nota-se que a estrutura E01, considerando o solo PE como subleito, sofreu a menor deformação de tração na base do revestimento, o que indica uma rigidez superior da estrutura dimensionada com esse material. Entretanto, como mencionado anteriormente, ao se optar por utilizar os módulos de resiliência não lineares, a resposta da estrutura será em função do estado de tensões atuantes. No caso das estruturas E02 e E03, dimensionadas respectivamente para os solos TR e RS, isentas da camada de sub-base, as tensões no topo do subleito são superiores, o que explica o decréscimo na rigidez desses materiais e as elevadas deformações de tração na fibra inferior do revestimento.

Em todos os casos, o número de repetições de cargas admissíveis à fadiga $\left(\mathrm{N}_{\mathrm{FADIGA}}\right)$ foi inferior ao número de solicitações previsto em projeto. Isso indica que o sistema irá sofrer ruptura, devido ao trincamento por fadiga, antes do tempo previsto em projeto. Essa constatação deixa evidente a importância do módulo de resiliência, em especial do solo empregado no subleito, no comportamento mecânico do sistema pavimento. BASTOS [9], ao analisar a estrutura de pavimentos dimensionados pelo método do DNIT considerando dois solos de subleito do Ceará, por meio da mesma ferramenta, obteve resultados semelhantes, com deterioração prematura dos pavimentos atribuída à fadiga.

De modo a reduzir as deformações de tração na base do revestimento, as espessuras de concreto asfáltico foram redimensionadas, e os números de solicitações à fadiga foram recalculados, sendo apresentados na Tabela 4. Como esperado, quanto menor a deformação de tração inicial, menor foi o acréscimo na espessura do revestimento. Esse aumento variou de $3,5 \mathrm{~cm}$ a $5,5 \mathrm{~cm}$, resultando em acréscimos na ordem de $35 \%$ a $55 \%$ da espessura inicial. De modo geral, com o aumento da espessura da camada de revestimento ocorreu um decréscimo nas deformações de tração na fibra inferior do revestimento e consequentemente um acréscimo no número de solicitações admissíveis ao trincamento por fadiga, sendo superior ao $\mathrm{N}_{\text {PROJETO}}$.

Tabela 4: Resultados obtidos no AEMC e aplicados ao modelo de desempenho a fadiga do FHWA

\begin{tabular}{ccccccc}
\hline & & \multicolumn{2}{c}{ ESTRUTURA INICIAL } & \multicolumn{3}{c}{ ESTRUTURA REDIMENSIONADA } \\
\cline { 3 - 6 } SOLOS & $\mathbf{N}_{\text {PROJETO }}$ & $\varepsilon_{\mathrm{t}}$ & $\mathbf{N}_{\text {FADIGA }}$ & $\varepsilon_{\mathrm{t}}$ & $\mathbf{N}_{\text {FADIGA }}$ & $\begin{array}{c}\text { ESPESSURA DO } \\
\text { REVESTIMENTO (cm) }\end{array}$ \\
\hline Solo CB - E01 & & $3,37 \mathrm{E}-04$ & $5,13 \mathrm{E}+06$ & $2,06 \mathrm{E}-04$ & $2,89 \mathrm{E}+07$ & 15,5 \\
Solo PE - E01 & $2,50 \mathrm{E}+07$ & $2,92 \mathrm{E}-04$ & $8,49 \mathrm{E}+06$ & $2,13 \mathrm{E}-04$ & $2,57 \mathrm{E}+07$ & 13,5 \\
Solo TR - E02 & & $3,06 \mathrm{E}-04$ & $7,20 \mathrm{E}+06$ & $2,11 \mathrm{E}-04$ & $2,66 \mathrm{E}+07$ & 14,0 \\
Solo RS - E03 & & $3,62 \mathrm{E}-04$ & $3,99 \mathrm{E}+06$ & $2,13 \mathrm{E}-04$ & $2,57 \mathrm{E}+07$ & 15,5 \\
\hline
\end{tabular}

Em ambas análises (AEMC e AASHTOWare) foi possível comprovar a importância do módulo de resiliência do solo de subleito no comportamento e no desempenho dos pavimentos ao longo dos anos. Constatou-se ainda a ineficácia do atual método de dimensionamento em vigor no Brasil, já verificada anteriormente por BASTOS [9], SANTOS et al. [15] e MOTTA [24]. Sob a perspectiva de mudança desse panorama, após anos de estudos, o novo método de dimensionamento de pavimentos flexíveis nacional - MeDiNa encontra-se em estágio final de elaboração, baseado em preceitos da abordagem mecanístico-empírico que considera as características resilientes de todos os materiais empregados no dimensionamento do pavimento.

\subsection{Resistência ao cisalhamento}

No que tange à resistência ao cisalhamento, as análises mecanicistas foram realizadas com o intuito de avaliar as tensões cisalhantes que ocorrem em campo para determinado solo e posteriormente comparar com as envoltórias de resistência obtidas em laboratório. Para tanto, os solos em estudo foram avaliados como material de fundação das estruturas dimensionadas e como subleito de pavimentos delgados compostos somente por tratamento superficial duplo (TSD).

Na Figura 3 é possível observar os gráficos da tensão cisalhante versus profundidade, para cada solo definido nesta pesquisa, considerando a primeira condição de análise. A simulação foi realizada em toda a estrutura, porém os gráficos apresentam somente as tensões de cisalhamento atuantes no subleito. Vale ressaltar que a presente análise foi realizada para três níveis de carregamento: ESRD com carga de 8,2 toneladas, 10 toneladas e 12 toneladas. 
Tensão Cisalhante $(\mathrm{kPa})$

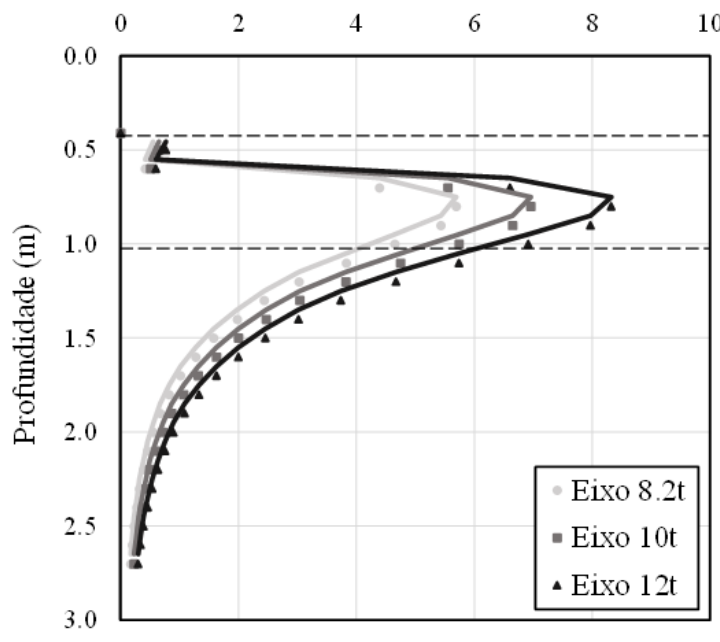

(a) Solo PE

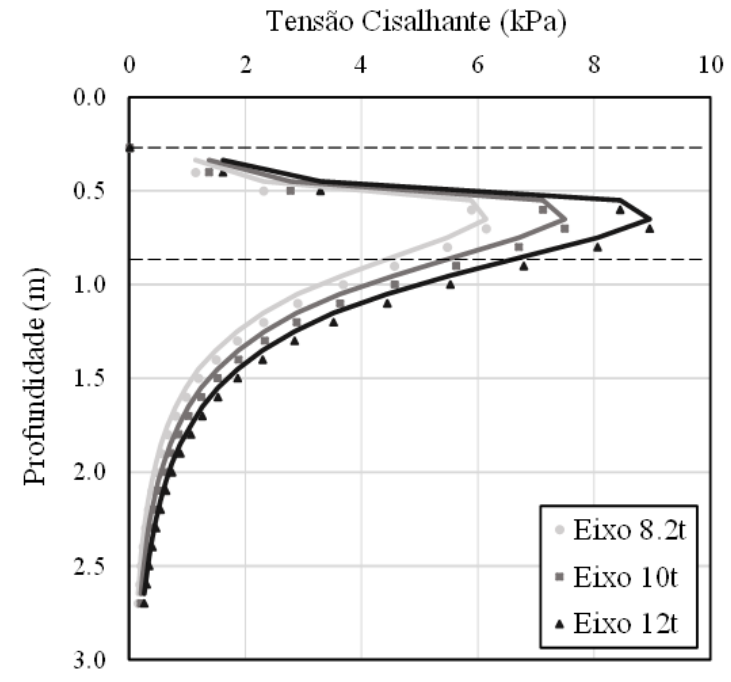

(c) Solo TR

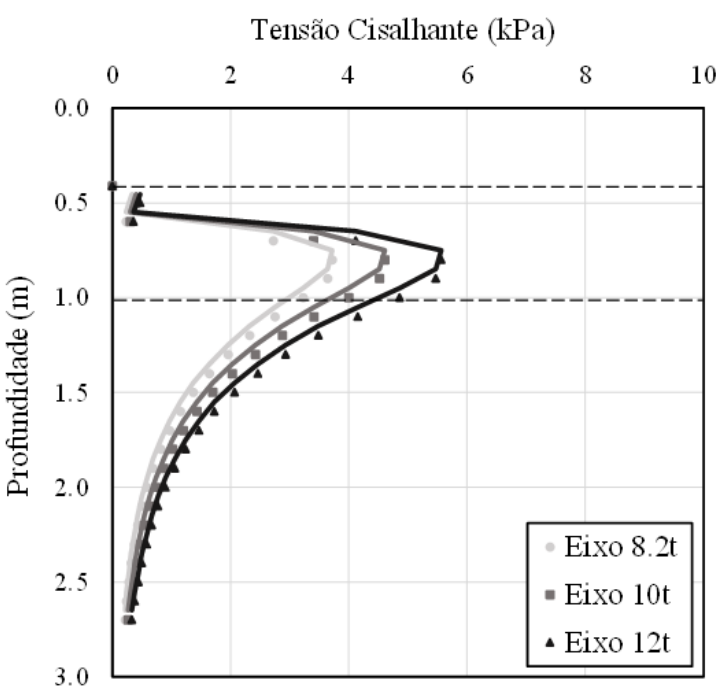

(b) Solo CB

Tensão Cisalhante $(\mathrm{kPa})$

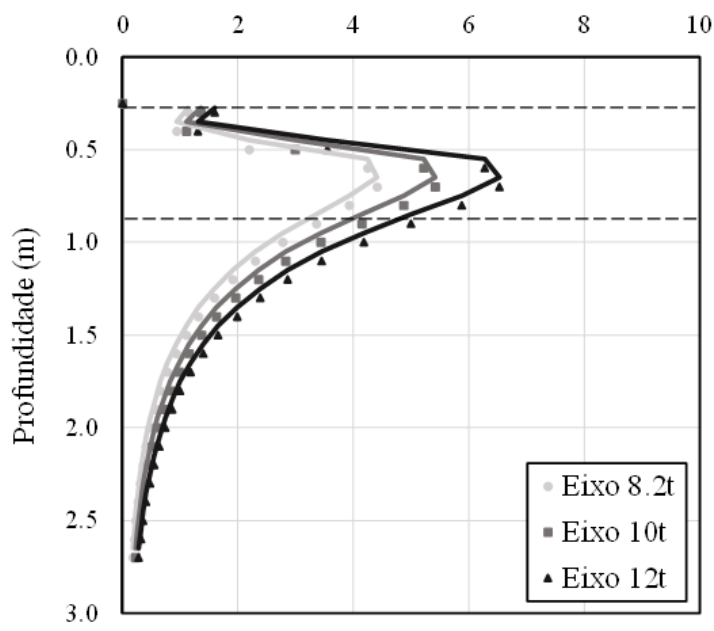

(d) Solo RS

Figura 3: Gráfico da tensão de cisalhamento versus profundidade para o solo PE (a); solo CB (b); solo TR (c); solo RS (d)

Analisando os gráficos apresentados, nota-se em um primeiro momento um acréscimo da tensão cisalhante com a profundidade. Esse acréscimo se dá até certo valor, onde a partir dele as tensões apresentam decréscimo com o aumento da profundidade, até próximo à tensão de cisalhamento igual à zero. Comportamento semelhante foi observado por SU et al. [25], na avaliação da dissipação das tensões cisalhantes em camadas asfálticas. Outro fato observado é que os valores máximos obtidos para as tensões cisalhantes ocorrem nos últimos $60 \mathrm{~cm}$ da terraplanagem, na chamada camada final de terraplanagem (CFT), destacada pelas linhas em tracejado.

A Figura 4 apresenta as envoltórias de ruptura obtidas nos ensaios de cisalhamento direto, para as condições ótima e úmida (wótima $+2 \%$ ), bem como os pares de tensões (cisalhante e normal) atuantes no subleito das estruturas, para cada nível de carregamento adotado, levando em consideração os quatro solos empregados nessa camada.

Em todos os casos, os pares de tensões atuantes foram muito baixos, inferiores aos valores apresentados nas envoltórias, não ocorrendo assim ruptura por cisalhamento dos solos. É possível observar que para baixas tensões normais, inferiores à $50 \mathrm{kPa}$, as tensões cisalhantes praticamente não variam com o acréscimo do carregamento. Para tensões normais verticais maiores, há um aumento da tensão de cisalhamento, conforme as cargas aplicadas aumentam. Em alguns casos, esse aumento chega a $30 \%$. 


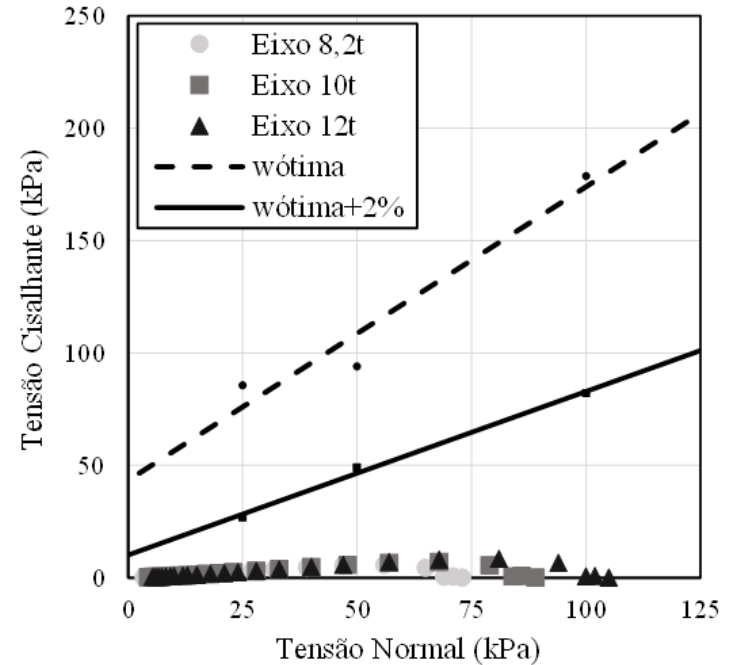

(a) Solo PE

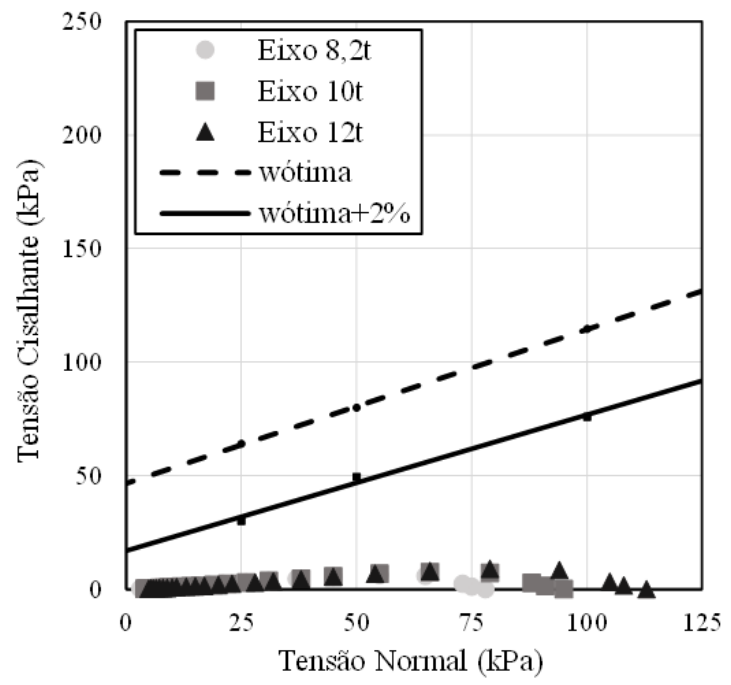

(c) Solo TR

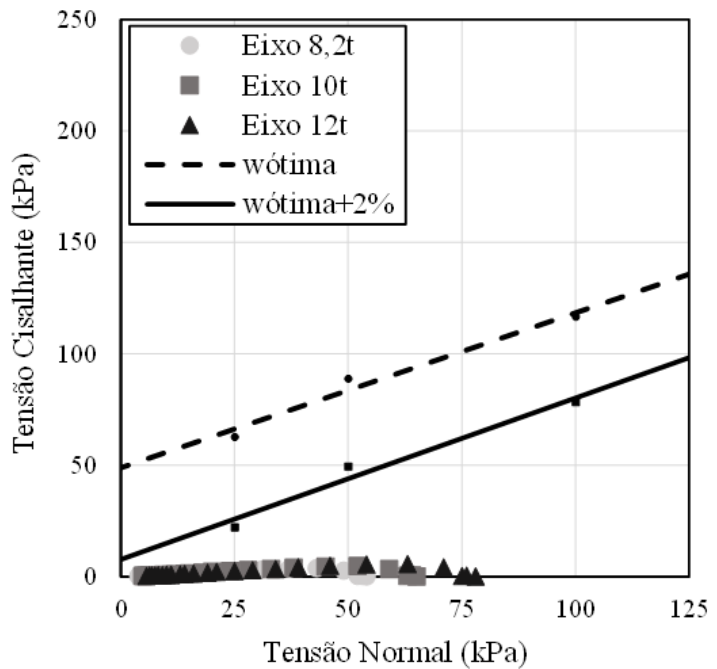

(b) Solo CB

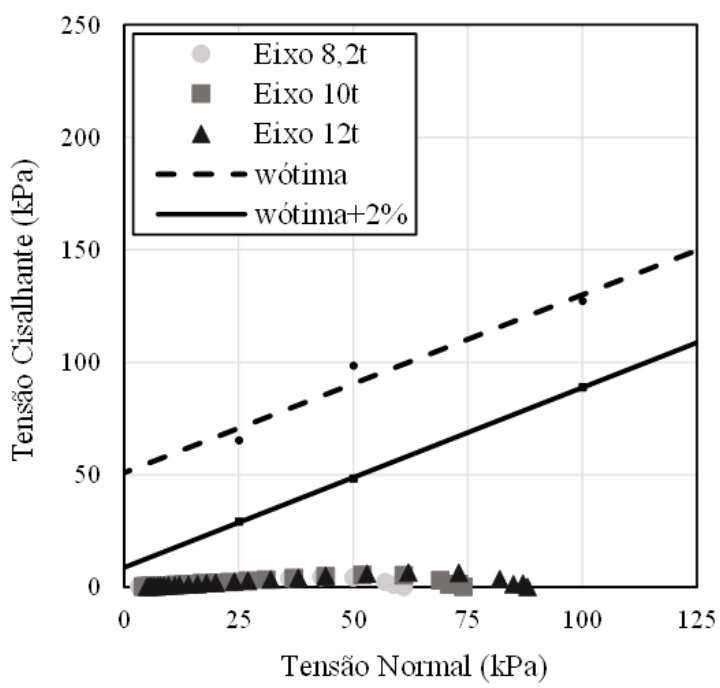

(d) Solo RS

Figura 4: Comparação entre as envoltórias de ruptura obtidas através do ensaio de cisalhamento direto (SANTOS et al. [15]) e as tensões atuantes nas estruturas dimensionadas para o solo PE (a); solo CB (b); solo TR (c); solo RS (d)

Dentre os solos estudados, o solo TR, empregado na estrutura E02, apresentou as maiores tensões de cisalhamento atuantes, em todos os níveis de carregamento. Os solos CB e RS apresentaram um comportamento semelhante nas simulações numéricas. Porém, segundo os resultados obtidos na ferramenta AEMC, o solo CB é o material que sofre as menores tensões cisalhantes em sua estrutura. O solo $\mathrm{PE}$ apresentou tensões cisalhantes atuantes intermediárias, quando comparado com os demais materiais, porém valores ainda distantes da ruptura por cisalhamento.

No que diz respeito à utilização dos solos como subleito de pavimentos de baixo volume de tráfego, as tensões de cisalhamento atuantes obtidas pela ferramenta AEMC apresentaram comportamento peculiar, como é possível visualizar na Tabela 5. Nesse caso, as tensões de cisalhamento máximas, assim com as tensões normais atuantes decrescem com o aumento do carregamento. A ênfase na Figura 5, dada pelo círculo em azul, ajuda a compreender essa singularidade. Por outro lado, com o acréscimo de carga a profundidade de propagação dessas tensões também aumenta, ou seja, os esforços provenientes do carregamento necessitam percorrer uma maior profundidade para que as tensões de cisalhamento se tornem nulas. Observou-se também que os resultados independem do tipo de material, dependendo somente do nível de carregamento, evidenciando que, nesse caso, as características elásticas não apresentam influência nas tensões atuantes.

Devido a esse comportamento peculiar, optou-se por utilizar as equações presentes em MEDINA e MOTTA [3] apresentadas na metodologia. Os valores de tensão cisalhante máxima, profundidade e tensão normal vertical no subleito obtidos por meio das equações são apresentados na Tabela 5. Vale salientar que essa análise foi realizada com a finalidade única de comparação com os resultados obtidos por meio da ferramenta AEMC. 
Tabela 5: Valores de tensão cisalhante máxima, tensão normal atuante e profundidade do pico de tensão de cisalhamento para cada nível de carregamento obtidos no AEMC e através das equações presentes em MEDINA e MOTTA [3]

\begin{tabular}{ccccccc}
\hline \multirow{2}{*}{$\begin{array}{c}\text { CARREG. } \\
(\mathbf{t})\end{array}$} & $\begin{array}{c}\text { TENSÃO } \\
\text { CISALHANTE } \\
\text { MÁXIMA (kPa) }\end{array}$ & $\begin{array}{c}\text { TENSÃO } \\
\text { NORMAL (kPa) }\end{array}$ & $\begin{array}{c}\text { PROF. } \\
\mathbf{( c m )}\end{array}$ & $\begin{array}{c}\text { TENSÃO } \\
\text { CISALHANTE } \\
\text { MÁXIMA (kPa) }\end{array}$ & $\begin{array}{c}\text { TENSÃO } \\
\text { NORMAL } \\
(\mathbf{k P a})\end{array}$ & $\begin{array}{c}\text { PROF. } \\
\mathbf{( c m})\end{array}$ \\
\hline 8,2 & 165 & 556 & 2 & & 457,6 & 7,44 \\
10 & 144 & 552 & 3 & 168 & 457,5 & 8,22 \\
12 & 111 & 546 & 4 & & 457,7 & 9,00 \\
\hline
\end{tabular}

Analisando os resultados é possível verificar a semelhança entre a tensão cisalhante máxima obtida no AEMC, para o carregamento de 8,2 t, referente ao eixo padrão rodoviário, e a tensão de cisalhamento máxima calculada por meio das equações apresentadas. Como a equação para obtenção da tensão cisalhante leva em consideração somente a pressão da área circular carregada e o coeficiente de Poisson do material em questão, não há diferença desse valor em relação ao nível de carregamento. A profundidade de ocorrência do pico de tensão cisalhante é superior aos valores obtidos no AEMC, mas ainda assim, seguem o mesmo comportamento, aumentando com o acréscimo de carga.

A Figura 5 apresenta além das envoltórias nas condições ótima e úmida, os pares de tensão de cisalhamento e tensão normal obtidos para os três níveis de carregamento através do AEMC e o valor de tensão cisalhante máxima determinado por meio das equações anteriormente citadas, representado no gráfico pelo ponto em vermelho. Constata-se que, apesar das tensões cisalhantes atuantes serem superiores àquelas obtidas para as estruturas dimensionadas pelo método do DNIT, principalmente em função do pavimento ser delgado, ainda se encontram abaixo das tensões cisalhantes máximas para qualquer uma das condições de umidade analisadas. Logo, afasta-se qualquer possibilidade de ruptura por cisalhamento dos solos em estudo utilizados na fundação de pavimentos de baixo volume de tráfego. Contudo, não se pode ignorar a possibilidade de ruptura por cisalhamento caso ocorram maiores oscilações de umidade ou até mesmo a saturação dos solos.

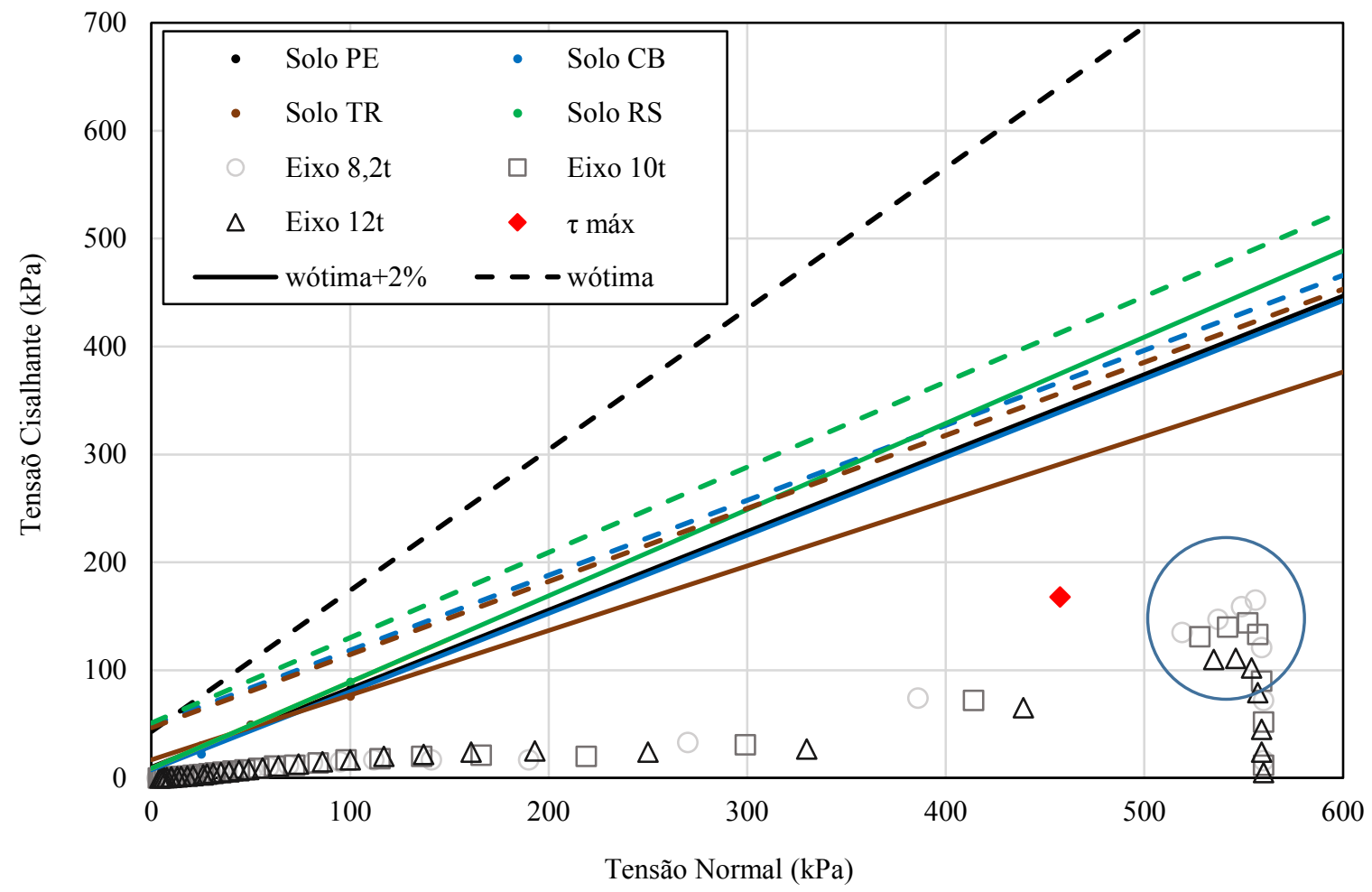

Figura 5: Comparação entre as envoltórias de ruptura obtidas através do ensaio de cisalhamento direto (SANTOS et al. [15]) e as tensões atuantes nos pavimentos de baixo volume de tráfego considerando os diferentes solos.

De modo a confirmar a inexistência de ruptura por cisalhamento, foram calculados ainda fatores de segurança, com base nas máximas tensões de cisalhamento atuantes, para cada nível de carregamento analisado, e nas tensões máximas cisalhantes extraídas das envoltórias de resistência das duas condições de umidade em estudo. Os resultados são apresentados 
na Tabela 6. Os fatores de segurança obtidos para a condição úmida (wótima $+2 \%$ ) foram inferiores àqueles obtidos para a condição de umidade ótima. Semelhante às análises apresentadas anteriormente, o solo TR apresentou o pior desempenho, comprovado pelos fatores de segurança inferiores em ambas condições. Considerando um fator de segurança variando de 1,4 a 1,5, adequado a obras viárias [26], constata-se que os quatro solos em estudo apresentam bom desempenho em ambas condições de umidade analisadas, assim como para os três níveis de carregamento adotados. Não obstante, há de se mencionar que os esforços provenientes do tráfego de veículos induzem à deformações plásticas e resilientes, diretamente relacionadas à ocorrência de afundamentos em trilhas de roda (ATR's) e a danificação por fadiga. Assim, nesse caso, o fator de segurança funciona como um parâmetro balizador do comportamento global do solo na estrutura, não substituindo, contudo, a verificação dos efeitos da deformabilidade desse material no desempenho do pavimento como um todo associado a uma análise probabilística.

Tabela 6: Fatores de Segurança para as condições ótima e úmida (wótima $+2 \%$ )

\begin{tabular}{|c|c|c|c|c|c|c|}
\hline SOLO & $\begin{array}{c}\text { CARGA } \\
(t)\end{array}$ & $\begin{array}{c}\text { TENSÃO } \\
\text { CISALHANTE } \\
\text { MÁX. (kPa) } \\
\end{array}$ & $\begin{array}{c}\text { TENSÃO } \\
\text { CISALHANTE } \\
\text { MÁX. wótima (kPa) }\end{array}$ & $\begin{array}{c}\text { TENSÃO } \\
\text { CISALHANTE MÁX. } \\
\text { wótima }+2 \%(\mathrm{kPa})\end{array}$ & $\begin{array}{c}\text { FS } \\
\text { wótima }\end{array}$ & $\begin{array}{c}\text { FS } \\
\text { wótima } \\
+2 \% \\
\end{array}$ \\
\hline \multirow{3}{*}{ Solo PE } & 8,2 & 165 & 769,5 & 414,9 & 4,7 & 2,5 \\
\hline & 10 & 144 & 764,3 & 411,9 & 5,3 & 2,9 \\
\hline & 12 & 111 & 756,4 & 407,6 & 6,8 & 3,7 \\
\hline \multirow{3}{*}{ Solo CB } & 8,2 & 165 & 435,3 & 410,8 & 2,6 & 2,5 \\
\hline & 10 & 144 & 432,5 & 407,9 & 3,0 & 2,8 \\
\hline & 12 & 111 & 428,4 & 403,5 & 3,9 & 3,6 \\
\hline \multirow{3}{*}{ Solo TR } & 8,2 & 165 & 423,3 & 349,9 & 2,6 & 2,1 \\
\hline & 10 & 144 & 420,6 & 347,5 & 2,9 & 2,4 \\
\hline & 12 & 111 & 416,5 & 343,9 & 3,8 & 3,1 \\
\hline \multirow{3}{*}{ Solo RS } & 8,2 & 165 & 490,5 & 453,5 & 3,0 & 2,7 \\
\hline & 10 & 144 & 487,4 & 450,3 & 3,4 & 3,1 \\
\hline & 12 & 111 & 482,6 & 445,5 & 4,3 & 4,0 \\
\hline
\end{tabular}

De modo geral, conclui-se que os solos em estudo não apresentam indícios de ruptura por cisalhamento, seja no subleito de uma rodovia ou na fundação de um pavimento de baixo volume de tráfego, tipicamente utilizados em vias urbanas ou estradas vicinais. Isso, entretanto não afasta a hipótese de que os mesmos sofram altas deformações plásticas, frisando novamente a importância do estudo do comportamento dos solos frente à deformação permanente.

\section{CONCLUSÕES}

Este trabalho teve como objetivo avaliar o desempenho de quatro solos de subleitos utilizados em importantes rodovias no estado do Rio Grande do Sul. Para tanto, avaliou-se a resposta mecânica de um pavimento padrão através de dois softwares, AEMC e AASHTOWare Pavement ME Design, relacionando-a com as características resilientes e de resistência ao cisalhamento dos solos em estudo, consideradas importantes propriedades geotécnicas em termos de obras rodoviárias.

Tanto as análises realizadas no AASHTOWare, quanto as análises realizadas no AEMC, evidenciaram a ruptura precoce do pavimento, independentemente do tipo de solo utilizado na fundação da estrutura. Concluiu-se que o excesso de trincas por fadiga é o mecanismo de ruptura predominante, o que está associado ao fato do atual método de dimensionamento - método do DNIT - não considerar o módulo de resiliência dos materiais de pavimentação no seu dimensionamento.

Constatou-se ainda que a resistência ao cisalhamento é uma propriedade de relevância secundária no que tange pavimentos flexíveis. Na fundação de pavimentos delgados, essa propriedade apresentou-se mais relevante, resultando em maiores tensões de cisalhamento atuantes. Entretanto, não houve indícios de ruptura por cisalhamento dos solos em nenhum dos casos analisados, o que não afasta, porém, a hipótese de altas deformações plásticas. Isso reforça a necessidade de maiores estudos relacionados a essa parcela da deformabilidade, principalmente em face ao novo método de dimensionamento nacional - MeDiNa, que levará em consideração além das características resilientes, o comportamento dos materiais acerca da deformação permanente.

\section{AGRADECIMENTOS}

Os autores agradecem a ANP/Petrobras pelo suporte fornecido através do projeto Rede Temática de Asfaltos; e ao CNPQ, Fapergs e Capes pelo apoio financeiro concedido por meio de bolsas de mestrado e produtividade em pesquisa. 


\section{BIBLIOGRAFIA}

[1] BALBO, J. T., Pavimentação asfáltica: materiais, projetos e restauração, 1. ed., São Paulo, Oficina de Textos, 2007.

[2] BERNUCCI, L., MOTTA, L. M. G., CERATTI, J. A. P., el al. Pavimentação Asfáltica: Formação Básica para Engenheiros, 1 ed., Rio de Janeiro, ABEDA, 2010.

[3] MEDinA, J., MOTTA, L. M. G., Mecânica dos Pavimentos, 3 ed., Rio de Janeiro, Editora Interciência, 2015.

[4] SALOUR, F., Moisture influence on structural behaviour of pavements: Field and Laboratory Investigations, Doctoral Thesis, Royal Institute of Technology, Stockholm, Sweden, 2015.

[5] LEKARP, F., RICHARDSON, I. R., DAWSON, A., "Influences of permanent deformation behavior of unbound granular materials", Transportation Research Record, v. 1547, n. 1, pp. 68-75, 1996. DOI: 10.1177/0361198196154700110

[6] MEDINA, J., “Mecânica dos Pavimentos: Aspectos Geotécnicos”, Solos e Rochas, v. 29, n. 2, pp. 137-158, 2006.

[7] SILVA, C. F. S. C., Análise de tensões em pavimentos a partir de modelo fisico instrumentado, Dissertação de Mestrado, COPPE/UFRJ, Rio de Janeiro, RJ, Brasil, 2014.

[8] FRANCO, F. A. C. P., Método de dimensionamento mecanístico-empírico de pavimentos asfálticos - SisPav, Tese de Doutorado, COPPE/UFRJ, Rio de Janeiro, RJ, Brasil, 2007.

[9] BASTOS, J. B. S., Influência da variação da umidade no comportamento de pavimentos da região metropolitana de Fortaleza, Dissertação de Mestrado, Universidade Federal do Ceará, Fortaleza, CE, Brasil, 2013.

[10] ERLINGSSON, S., AHMED A. W., "Fast layered elastic response program for the analysis of flexible pavement structures", Road Materials and Pavement Design, v. 14, n. 1, pp. 196-210, 2013. DOI: 10.1080/14680629.2012.757558

[11] GHOSH, A., PADMAREKHA, A., KRISHNAN, J. M., "Implementation and proof-checking of mechanistic-empirical pavement design for Indian highways using AASHTOWare Pavement ME Design software", Procedia Social and Behavioral Sciences, v. 104, pp. 119-128, 2013. DOI: 10.1016/j.sbspro.2013.11.104

[12] LEE, K.-W.W., WILSON, K., HASSAN, S. A., "Prediction of performance and evaluation of flexible pavement rehabilitation strategies", Journal of Traffic and Transportation Engineering (English Edition), v. 4, n. 2, pp. 178-184, 2017. DOI: $10.1016 /$ j.jtte.2017.03.005

[13] GUDIPUDI, P. P., UNDERWOOD, B. S., ZALGHOUT, A., "Impact of climate change on pavement structural performance in the United States", Transportation Research Part D: Transport and Environment, v. 57, pp. 172-184, 2017. DOI: 10.1016/j.trd.2017.09.022

[14] SPECHT, L. P., BABADOPULOS, L. F. A., DI BENEDETTO, H., el al."Application of the theory of viscoelasticity to evaluate the resilient modulus test in asphalt mixes", Construction and Building Materials, v. 149, pp. 648-658, 2017. DOI: 10.1016/j.conbuildmat.2017.05.037

[15] SANTOS, T. A., SPECHT, L. P., PINHEIRO, R. J. B., el al."Avaliação da resistência e da deformação resiliente de quatro solos de subleitos rodoviários no estado do Rio Grande do Sul”, TRANSPORTES, v. 27, n. 1, pp. 48-64, 2019. DOI: $10.14295 /$ transportes.v27i1.1531

[16] YODER, E. J., WITCZAK, M. W., Principles of Pavement Design, 2 ed., New York, John Wiley \& Sons Inc., 1975.

[17] SOUZA, M. L., Método de projeto de pavimentos flexíveis, 3 ed. rev. e atual, Rio de Janeiro, 1981.

[18] DNIT - Departamento Nacional de Infraestrutura de Transportes - IPR 719. Manual de Pavimentação, 3 ed, Rio de Janeiro, RJ, 2006.

[19] KLAMT, R. A., Influência da ação do tráfego no custo de construção e restauração de pavimentos asfálticos, Dissertação de Mestrado, Universidade Federal de Santa Maria, Santa Maria, RS, Brasil, 2014.

[20] NÚÑEZ, W. P., Análise experimental de pavimentos rodoviários delgados com basaltos alterados, Tese de Doutorado, Universidade Federal do Rio Grande do Sul, Porto Alegre, RS, Brasil, 1997.

[21] RIBAS, J., Parâmetros elásticos típicos de materiais de pavimentos flexíveis do Rio Grande do Sul e sua aplicação em uma análise de custo/benefício, Dissertação de Mestrado, Universidade Federal de Santa Maria, Santa Maria, RS, Brasil, 2014.

[22] MATTOS, J. R. G., Monitoramento e análise do desempenho de pavimentos flexíveis da ampliação da rodovia BR290/RS - A implantação do projeto Rede Temática de Asfalto no Rio Grande do Sul, Tese de Doutorado, Universidade Federal do Rio Grande do Sul, Porto Alegre, RS, Brasil, 2014. 
[23] HOLTZ, R. D., KOVACS, W. D., An introduction to geotechnical engineering, 1 ed., New Jersey: Prentice-Hall, 1981.

[24] MOTTA, L. M. G., Método e Dimensionamento de Pavimentos Flexiveis: Critério de Confiabilidade e Ensaios de Cargas Repetidas, Tese de Doutorado, COPPE/UFRJ, Rio de Janeiro, RJ, Brasil, 1991.

[25] SU, K., SUN, L., HACHIYA, Y., MAEKAWA, R., "Analysis of shear stress in asphalt pavements under actual measured tire-pavement contact pressure", In: Proceedings of the 6th ICPT, pp. 11-18, Japan, 2008.

[26] DNER - Departamento Nacional de Estradas de Rodagem. DNER PRO 381: Projeto de aterros sobre solos moles para obras viárias, Rio de Janeiro, RJ, 1998.

\section{ORCID}

Thaís Aquino dos Santos

Rinaldo José Barbosa Pinheiro

Luciano Pivoto Specht

Lélio Antônio Teixeira Brito https://orcid.org/0000-0001-6327-3776

https://orcid.org/0000-0003-1444-9493

https://orcid.org/0000-0002-8709-6273

https://orcid.org/0000-0002-7774-2948 